\title{
Commission 44: Space \& High Energy Astrophysics
}

\author{
PRESIDENT: Haruyuki Okuda \\ VICE-PRESIDENT: Gunther Hasinger \\ BOARD: M. D. Arnaud, S. Bludman, J. Braga, N. Brosch, \\ L. Gurvits, H. Hasan, G. Helou, I. Howarth, H. Inoue, \\ P. Luigi, and G. Srinivasan
}

\section{Introduction}

Division XI was born by merging Commission 44 "Space and High Energy Astrophysics" and Commission 48 "High Energy Astrophysics" by the decision at the IAU General Assembly in The Hague (1994). As the naming of space astronomy is technique oriented, i.e. astronomy from space, it covers quite a wide range of astronomy, almost all branches of astronomy are included by the progress of space observations. Historically, it started from high energy astronomy, UV, X, and gamma rays astronomy, somewhat including cosmic ray physics. However, in these days, space observations have expanded to low energy astronomy, such as optical, infrared, submillimeter and even radio waves(Space VLBI). The addition of space astrophysics, thus, has made it rather complicated and difficult to organize the Division activities. When it was concerned with the high energy astrophysics, relevant sciences had much commonality and the used techniques were similar, mostly coming from particle physics and the observations are made exclusively in space. On the other hand, astronomy in low energies, optical, infrared/submillimeter and radio waves, were originated from mostly ground based observations, therefore, the classification is more or less not technique oriented, but subject oriented, as solar, stellar or galactic astronomy etc. They have less commonality among each other and particularly with the high energy astrophysics.

This situation is also reflected in the Division membership, which is quite large, but the majority of the members are high energy astronomers, UV, X-ray and gamma ray and some cosmic ray astrophysicists. Members of optical, infrared/submillimeter and radio astronomers are relatively few, most of the relevant astronomers have chosen their Division by subjects of their specialties. Many of the registered astronomers have been concerned with cosmic ray physics or particle physics in the past, by the historical fact that the new astronomies have been initiated by them.

This situation has raised some problems, e.g. in the proposals for the IAU meetings and their support and coordination. Some meetings are proposed on the basis of the technical background and others based on their subjects. Similar proposals are submitted independently by different Divisions without sufficient communication among them. The problem is more serious in the low energy astrophysics, by its traditional reason as mentioned.

Scientifically, the Division XI is enjoying very exciting and prosperous years, many missions in a variety of fields are in orbit and being operated actively. Many new missions are also in preparation and waiting for launches in the near future. The missions cover almost the whole range of wavelengths, from radio astronomy to gamma ray astronomy, as well as even the missions related to particle astrophysics and gravitational wave 
detections. Their products are enormous and many exciting discoveries are revolutionizing our concepts of the Universe. It is beyond the author's scope to cover them completely, so only a brief report on major activities will be given with helps of the board members.

\section{UV, X-ray and gamma ray astronomy}

\subsection{AXAF-Chandra}

Two big X-ray missions, AXAF-Chandra and XMM-Newton, were launched successively in 1999 and are continuing their operation actively. They have complementary functions; Chandra has an extremely high angular resolution like an optical telescope, while Newton has a large collecting power favorable to spectroscopic measurements. With their large collecting power of photon and high sensitivity detectors, they have expanded the observations to almost all kind of astronomical objects, from comets, planets to the distant quasars of cosmological importance.

With its unprecedented image quality and high sensitivity, Chandra has made extensive and deep surveys of many kinds, which have led to many discoveries of great interest. The followings are only some typical examples. It has been found in the deep survey of distant galaxies that the X-ray background radiation is resolved into discrete sources, mostly of super massive black holes associated with AGNs. In the GOODS project, super massive black holes embedded in dusty young galaxies were found in the Lockman Hole area. Chandra has provided a new line of investigation of dark mass estimation in clusters of galaxies by observing hot gas emitting X-ray. A gigantic hot gas ejection produced by a super massive black hole was observed in the galaxy cluster, MS0735.6 + 7421. Many intermediate mass black holes, characterized by ultra luminous X-ray emission were found in M 74. As for Galactic sources, a swarm of medium size black holes has been found in the Galactic center region and an X-ray source has been identified to Sgr A*, which shows flare activity. Large numbers of young star objects are present in star forming regions and they show violent X-ray flare events. It should be stressed that the high resolution observations are essential for identification of the sources to the objects observed in other wavelengths. Chandra is also very powerful in uncovering fine details of morphological structures of sources. The beautiful pictures of the Crab pulsar rings and jets, the shockwaves and jets in the Cas A supernova remnant, the compact X-ray source identified with Sgr A* are good examples.

\subsection{XMM-Newton}

XMM-Newton, having extended sensitivity to high energy and good spectroscopic capability, has provided interesting results. It has made the first detection of resonant cyclotron absorption in an isolated neutron star, 1E1207.4-5209. A large and anomalous line broadening of $\mathrm{K} \alpha$ fluorescent iron line was observed in the AGN MCG 6-3-15 by ASCA, which has been beautifully confirmed by XMM-Newton with its better sensitivity and resolution. The line profile is explained by the combination of Doppler and gravitational redshift of the line emitted in an accretion disc near the event horizon of a black hole. The gas in such a region should fall into the black hole with relativistic velocity and be put in the enormously strong gravitational field. However, it is intriguing that the relativistic distortion has not been found in the majority of AGNs in the large sample observed by XMM-Newton. On the other hand, a relativistic Fe K $\alpha$ line has been detected by XMM-Newton in the average spectrum of the X-ray background sources. A very interesting spectral modulation of the $\mathrm{Fe} \mathrm{K} \alpha$ line has been found in the XMM-Newton spectra of the Seyfert galaxy NGC 3516. There is a systematic variation of the flux at intervals of $25 \mathrm{ksec}$, while the spectral peak moves in energy between 5.7 
and $6.5 \mathrm{keV}$. This modulation can be explained if the emission arises from a spot on the accretion disc at between 3.5 and 8 Schwarzshild radii.

Meanwhile, the observable distance of the luminous AGN is now reaching the edge of the Universe, or very early phase of galaxy formation, a quasar with $\mathrm{z}=5.8$ has been detected. XMM-Newton observations of clusters of galaxies have not detected the cool gas in the centers of galaxies expected by the isobaric cooling flow model. In the meantime researchers are of the opinion that energy feedback from the central super-massive black hole prevents the gas from cooling.

Besides these observations, XMM-Newton has made important contribution for the Galactic objects. X-ray activity in the Galactic center is intriguingly quiet in spite of the presence of super massive black hole. Chandra detected first an X-ray flare event at the position of Sgr A*, while XMM-Newton observed the brightest flare in 2002. All these observations have made the black hole hypothesis of Sgr A* quite convincing, together with the infrared observations of stars orbiting around Sgr $A^{*}$. In the field of gamma ray bursts, the concentric ring like structure around the gamma ray burst GRB 031203 is one of the most fascinating discoveries. As for stellar phenomena, coronal activity has been found in solar type stars, one of which shows 8 years cyclic variation like the sun.

As is seen, the observations of the two facilities have been expanded to every kind of astronomical objects and every kind of astronomical phenomena. They are now used as general facilities competitive to ground base observatories. Many collaborative works have been done using their complementary functions. Wider collaborations with other facilities as HST and SST, as well as ground base observatories have been made successfully. The GOODS project, a deep survey of high red-shift objects was one of good examples. Most of the above achievements have been more or less cooperative endeavors between Chandra and XMM-Newton and their contribution are not rigorously separable.

\section{3. $S U Z A K U$}

Suzaku (Astro-E2) has been launched successfully in 2005. Apart from imaging telescopes with CCD detectors and a modern hard X-ray detector, it carries the first cryogenic bolometer experiment with an excellent spectral resolution $(7 \mathrm{eV})$ which was demonstrated for the first time in orbit. Unfortunately, after about 3 weeks of operation in orbit all the liquid helium in the cryostat was lost. The cause of this event is still being investigated. However, with the rest instruments, having its excellent CCD energy resolution in the soft $\mathrm{X}$-ray range, its comparatively low intrinsic background and its unprecedented sensitivity in the hard X-ray band, Suzaku now works as a powerful wideband X-ray observatory promising exciting results.

\subsection{INTEGRAL}

As for the observations in higher energy range, gamma ray, INTEGRAL launched in 2002 is working actively, providing new insight to understanding of the violent and exotic objects such as neutron stars, black holes, AGNs and particularly mysterious gamma ray bursts activities. It is equipped with an optical camera and X-ray detector, which enable simultaneous observations of importance for identification and physical interpretation. With the coverage of high energy detection up to $10 \mathrm{MeV}$, it has a unique capability for the detection of nuclear lines. Among the most exciting early results is the detection of a strong $511 \mathrm{keV}$ annihilation line from the Galactic center, the study of the Galactic Ridge emission in the hard X-ray band and the discovery of several heavily obscured galactic $\mathrm{X}$-ray sources. The intensity distribution of $1.8 \mathrm{MeV}$ line of the radio active isotope ${ }^{26} \mathrm{Al}$ has been also observed. It is useful for study of nuclear synthesis in the Galaxy. This 
presence of such line emission means that active acceleration of high energy particles is still going on in the central region of the Galaxy. A recent observation has confirmed the discovery by ASCA of the Fe fluorescent emission from the giant molecular cloud Sgr $\mathrm{B}_{2}$ located at 300 l.y. from the Galactic center. The emission is explained by the fluorescent line induced by energizing radiation produced by a violent explosion occurred in the Sgr $A^{*} 300$ years ago, very recently.

\subsection{AGILE and GLAST}

Two new missions for the high-energy gamma ray range are currently prepared for a launch in 2007. AGILE is a small Italian satellite, and GLAST a large NASA observatory. These missions will fill an important gap after the demise of the Compton Gamma Ray Observatory. In particular, GLAST, with its unprecedented collecting area and angular resolution will provide a completely new image of the Gamma sky.

\subsection{Swift, HETE}

After the successful launch and operation of HETE-2, which provides Gamma ray burst triggers on a regular basis, the field has been transformed by the launch of the Swift gamma ray burst mission. The Swift satellite carries the Burst Alert Telescope (BAT), the X-ray Telescope (XRT) and a UV-Optical Telescope (UVOT). After the discovery of a Gamma ray burst, Swift automatically slews to the direction of the burst and observes the burst and its afterglow with the narrow-field instruments. The XRT has been an especially effective instrument, showing new phenomena in the early evolution of Gamma ray burst afterglows and allowing a resolution of the problem of the origin of short, hard bursts.

\section{7. $G A L E X$}

The UV mission, GALEX (Galaxy Evolution Explorer), a successor of FUSE, are making extensive surveys of galaxies with its wide field of view UV cameras. With its complementary function to HST, the global structures of star formation activity in the galaxies are well contrasted to the general stellar activities.

\section{Optical, infrared/submillimeter and radio astronomy}

\subsection{HST}

Hubble Space Telescope was celebrated its 15th birthday in 2005. By the continuous restoration and addition of new instruments, as NICMOS and ACS, it is still playing major role in the recent progress of astronomy. With unprecedented sharpness, HST has provided beautiful pictures of planets, planetary nebulae and galaxies, by which detailed structures of planetary atmosphere, fine textiles of expanding gas of planetary nebulae, and activities of star formation in galaxies etc. Some of these characteristics had been glimpsed in the images taken by ground based telescopes, but it should be highly respected that the HST's images have confirmed the facts with no other possibility. With its unprecedented sensitivity and resolution enabled by the ideal observational conditions, HST has made the important observations such as the acceleration of the expanding Universe by observations of distant supernovae, the violent activities of initial phase of galaxy formation by the Ultra Deep Survey of Galaxies, the existence of extrasolar planets and the elucidation of the mysterious gamma ray bursts. The collaborative project with Chandra, XMM-Newton and ground based observatories, named GOODS, is on going for extensive deep galaxy survey. There are so many other achievements that 
it is hardly to mention them all. It is a great pity that the quit of the operation is anticipated by the withdrawal of the Space Shuttle program.

\subsection{JWST}

The introduction of adaptive optics and interferometric technique has made ground based telescopes competitive to HST in resolution, particularly in infrared observations. However, the superiority of the observational conditions is irreplaceable. This is the motivation of implementing a larger space telescope, so called JWST (formerly called as NGST), which is now under development and to be launched in 2010. No doubt, the new space telescope will open another new era of astronomy as the HST has done.

\subsection{SIRTF-SST}

Following the great success of the first observatory type infrared mission, ISO, a new infrared satellite SIRTF (Space InfraRed Telescope Facility) renamed as Spitzer Space Telescope (SST) after the launch in August 2003 is in full operation. Taking an Earth trailing solar orbit to avoid the heat of the Earth, SST enjoys the superb sensitivity with low background emission which allows it to detect the faintest or deepest objects ever. The first period of the mission contributed legacy programs with their enormous output data, so extensive, it is impossible to review them all. The deep galaxy survey made with higher sensitivity, in the 24 micron band in particular, has confirmed the active phase of star formation rate of galaxies in the past around $\mathrm{z}=1$ to 2 , as suggested in the ISO's observations. The observation limit has been greatly expanded now, reaching the initial phase of galaxy evolution, with several galaxies detected at $z>6$, with colors characteristic of mature stars; these are the earliest known galaxies today. The wide field of view and high resolution of the infrared cameras with large format arrays have enabled detailed morphological observations of Galactic nebulae as well as galaxies, by which understanding of the star formation activities both in the Galaxy and other galaxies has been greatly improved. A number of young stellar objects have been sampled, revealing evolutionary trends of the early phase of star formation. The global structure of star formation in other galaxies has been studied in detail by the improvements of sensitivity and resolution in both near and far infrared. The discovery of many low temperature and low mass objects has provided valuable materials for studies of brown dwarfs and particularly for identification of extra-solar planets. One of the most exciting result to date from SST has been the direct detection of emission from extra-solar planets at several wavelengths, allowing direct estimates of temperature and size. Spectroscopic capability in near and mid infrared ranges are actively used for studies of physics and chemistry in a variety of objects, stellar atmosphere, nebulous objects, and interstellar matters etc. The ubiquitous presence of ice and PAH materials has been shown. The SST has now become an indispensable facility playing a major role in astronomy. Many collaborative works have been organized with optical, $\mathrm{X}$ ray and radio missions, such as HST, Chandra, XMM-Newton as well as VLBI missions.

\subsection{Herschel}

The most frustrated thing in the infrared space missions have been the poor angular resolution in far infrared observations, which is limited by diffraction of the telescope apertures. This is due to the technical problem that large telescopes cannot be accommodated in vacuum chambers required for cryogenic cooling. The limitation is partially remedied by applying warm launch and radiation cooling in orbit. This method has been partially applied to the SST successfully by adopting the interplanetary orbit. Applying the same technique, a $3.5 \mathrm{~m}$ infrared telescope is under development for the Herschel 
mission to be launched in an L2 point in 2007 by ESA. This is a more advanced mission than the SST, equipped with sophisticated instruments including heterodyne receivers for submillimeter observations. A similar mission called SPICA is under planning in Japan. By using multi stage mechanical coolers, the whole telescope will be cooled to liquid He temperature without cryogen, that should afford the best performance in far infrared observations.

\subsection{SOFIA}

The atmospheric window for infrared observations is practically open at the stratospheric altitude, although still strong background emission is left in far infrared. A large aperture $(3 \mathrm{~m})$ infrared telescope called SOFIA, which is on board a jumbo jet Boeing 747, has been developed for years, and its first light was recently observed. The large aperture will provide observations with the highest resolution in far infrared. Its full commission is expected soon. Easy application of new technologies and easy procurements of new instruments are a great advantage for enabling observations with state of the art capabilities throughout the lifetime of the mission.

\subsection{SWAS, ODIN}

Even with small scale missions, SWAS and ODIN specialized for submillimeter observations with heterodyne receivers, have made unique contributions for the studies of interstellar and circumstellar environments by observing water and oxygen molecular transitions, which are inaccessible from the ground. Recently they participated in the Deep Impact program of Comet Tempel 1, and succeeded in detection of a violent water vapor ejection.

\subsection{ASTRO-F}

An advanced mission ASTRO-F specialized for general survey observations will be launched by ISAS/JAXA early in 2006. With the substantially better sensitivity, resolution and larger field of view than IRAS, it will make deep sky survey covering near, mid and far infrared ranges. It will provide a large and homogeneous data base useful for morphological and statistical studies of infrared sources as well as for serendipitous discoveries of exotic sources.

\subsection{WMAP}

As the first major cosmology mission, COBE took a great step towards establishing the Big Bang cosmology, with the perfect confirmation of the black body nature of the cosmic microwave background. It also gave evidence of anisotropy in the brightness distribution of the background radiation, although it was very subtle. The advanced mission WMAP, launched in 2001 as the first astronomical observatory at L2 point, has confirmed the COBE's results perfectly with the higher sensitivity and higher resolution. The excellent quality of the observational data has enabled detailed analysis of the power spectrum of the brightness fluctuation of small angular scales. Theoretical interpretation of the observed results has led to decisive estimation of the fundamental cosmological parameters; Hubble constant, flatness of space, as well as the contents of baryons, dark matter and dark energy in the Universe. These quantities were debated for decades, and WMAP has given the most precise answers to them so far available. Indeed, it was a historical mission for cosmology. By detailed analysis, however, there has been found a subtle but serious deviation in the power spectrum of spatial fluctuation at low angular frequencies, that is controversial to the inflation theory. The spacecraft is in good health and still accumulating the data to improve the accuracy of analysis, in particular promising improvements of polarimetric observations. 


\subsection{Planck}

As a successor of WMAP, an advanced mission called Planck is under development by ESA, CNES and ASI. Its improved capability of polarimetric measurement, together with the higher sensitivity and the finer angular resolution, will offer a crucial test for the inflation model of the early Universe. Detailed measurements of the Sunyaev-Zeldovich effect are also expected. Planck and Herschel will be launched by the same rocket Ariane $\mathrm{V}$ in 2007 or 2008.

\section{Others}

\subsection{General relativity, gravitational wave}

A gravity probe, GP-B, with very sophisticated gyroscopes floated in superfluid helium temperature was launched in 2004 for rigorous test of the two key predictions of general relativity theory, the geodesic effect and the frame dragging. The effects are extremely subtle and should be measured with extraordinary precision. Their results are waited with great anticipation. GP-B ran out of helium in October 2005.

As an advanced version of the ground base experiments, as TAMA-300, GEO-600 and LIGO, a space mission, LISA is under planning. It is a long span laser interferometer for detection of gravitational waves, to be launched to a Lagrangean point orbit.

\subsection{Ultra high energy cosmic rays}

There has been a long and controversial discussion on presence of ultra high energy cosmic rays, as high as $10^{19}$ to $10^{20} \mathrm{eV}$. A Space Station borne mission called as EUSO has been proposed as an international collaborative endeavor. The idea is to observe light flashes of fluorescent light induced by giant air showers by mounting a wide field camera onboard the Space Station. It is an ambitious mission, but there is some concern concerning the future prospect of the Space Station project itself.

\section{Summary}

The space missions have been expanded to almost every field of astronomy and relevant to every kind of astronomical objects or phenomena. Dozens of missions are now in orbits and another dozen of missions are under development, awaiting launches in coming years. These missions have explored and will solve the problems of the natures of neutron stars, black holes, super massive black holes haunting the Galactic center and AGN cores, far distant galaxies reaching to almost the edge of the Universe or the very instant of galaxy formation, as well as the origin of stars and planets. The golden age of space observations will continue and the advanced missions will provide great strides of progress, in a variety of fields of astronomy. A clear characteristics of these activities is that many of the missions are carried out by large consortia, with multi-discipline participants. Sometimes, collaborations are organized among multiple missions and with ground base observatories. The collaborations yield more valuable and comprehensive data sets and results. Many missions are international in hardware developments and data analysis. As the scale of missions becomes larger, international collaboration is indispensable to afford the high cost and work force needed. These developments should increase the importance of the activity of Division XI, but, at the same time, raise the complexity and difficulty of the activities. This is the issue which is mentioned in the chapter 1 . The solution may not be easily found, but the problem should be discussed elsewhere. 
In any case, the future of space astronomy is very promising and prosperous. Many big missions are on the horizon and they will certainly contribute to promote astronomy in various aspects, but it should be stressed that small missions are also very important to cultivate new fields. Beppo-SAX, HETE-2, and Swings are good examples.

\section{Working Groups}

There are two Working Groups in Division XI, "Particle Astrophysics" and "Astronomy from the Moon".

\subsection{Particle Astrophysics}

This Working Group has been set up to care the field concerned with cosmic rays, high energy gamma rays, neutrino astrophysics and gravitational wave detection, which have been traditionally operated from the ground. The progress in technology and infrastructure of space activities has opened the possibility of making heavy load experiments as of the above missions. The search for the dark matter has now become a great concern.

\subsection{Astronomy from the Moon}

This Working Group has been organized to explore the possibility of observations from the Moon. It has a big potentiality to make a various kind of observations, but, at the same time, it should become a big enterprise and need a wide range international collaboration. The Working Group should play an important role for such collaborations. Many issues such as astrophysical goals of the projects, relevant technical problems, coordination among the projects and international collaboration are to be investigated.

In both Working Groups, the chair persons have been changed, from Prof. Marco Salvati of the Arcetri Observatory to Prof. Reinhard Schlickeiser of Bochum University in the Working Group of Particle Astrophysics and from Prof. Norio Kaifu of National Astronomical Observatory of Japan to Prof. Sallie Baliunas of Havard Smithsonian Center of Astronomy in the Working Group of Astronomy from the Moon. Both Workings will have Science Business Meetings in the General Assembly in Prague.

\section{Acknowledgements}

The author is grateful to Gunther Hasinger, George Helou, Reinhard Schlickeiser and Sallie Baliunas for their helps in preparation of the report. 